\title{
Análise de referências utilizadas por pesquisadores na revista Gestão \& Produção
}

\author{
Analysis of references used by researchers \\ in the journal Gestão \& Produção
}

Fabiana Souza de ANDRADE'

Carlos Fernando JUNG²

\section{Resumo}

Este artigo apresenta os resultados de uma pesquisa descritiva, com abordagem quantitativa e estratégia documental, que teve a finalidade de efetuar uma análise das referências listadas nos artigos das publicações regulares da revista "Gestão \& Produção" através da adaptação dos conceitos da bibliometria. Considerando o objetivo da pesquisa, executou-se a análise das listas de referências, especificando-se: 1) como sujeitos investigados os documentos referenciados nos artigos, 2) como variáveis os elementos determinísticos obtidos através da análise de referências e 3) como tipo de recurso a pesquisa bibliográfica. Com base nesses parâmetros, analisaram-se os documentos utilizados no embasamento teórico dos artigos através da identificação, do mapeamento e da apuração quantitativa: a) dos itens listados em cada trabalho; b) da vida média da literatura; c) do idioma; d) da frequência de uso das referências e; e) da frente de pesquisa. Como resultado, identificaram-se os principais aspectos das fontes de pesquisas que fundamentam os trabalhos em Engenharia de Produção e suas subáreas.

Palavras-chave: Análise bibliométrica. Análise quantitativa. Estudo métrico. Produção científica. Referências.

\begin{abstract}
This article presents the results of a descriptive research with quantitative approach and strategy based on the documentary method, with the aim of analyzing the references listed in articles appearing in regular publications of the Journal "Gestão \& Produção" by adapting the concepts of bibliometric citation analysis. Given the objective of the research, an analysis was made of the reference lists specifying: 1) how subjects investigated the documents referenced in the articles, 2) as elements of deterministic variables obtained by analyzing references and, 3) as the type ofbibliographic research resource. Based on these parameters, documents were analyzed used in the theoretical basis of the articles by means of identification, mapping and quantitative determination: a) of the items listed in each study; $b$ ) the mean life of the literature; $c$ ) the language; $d$ ) the frequency of use of references and; e) research front. As a result, the main aspects of the sources of researches on which studies in Production Engineering and its sub areas are based were mapped.
\end{abstract}

Keywords: Bibliometric analysis. Quantitative analysis. Metrics study. Scientific production. References.

\section{Introdução}

Os primeiros estudos de análise de citação tiveram início em 1927, quando Gross e Gross (1927) propuseram- -se a tabular as referências do "The Journal of the American Chemical Society", com o objetivo de fornecer às bibliotecas um instrumento de previsão para a demanda bibliográfica de documentos na área de Química. Somente após quase

\footnotetext{
1 Mestranda, Universidade Federal do Rio Grande do Sul, Programa de Pós-Graduação em Engenharia de Produção. Av. Osvaldo Aranha, 99, Porto Alegre, RS, Brasil. Correspondência para/Correspondence to: F.S. ANDRADE. E-mail: <bia.souzandrade@gmail.com>.

2 Professor Doutor, Universidade Federal do Rio Grande do Sul, Programa de Pós-Graduação em Engenharia de Produção. Porto Alegre, RS, Brasil. Recebido em 5/7/2012, reapresentado no dia 27/8/2012 e aceito para publicação em 10/10/2012.
} 
três décadas, entretanto, os estudos de Garfield (1955) deram novo impulso aos trabalhos sobre a técnica.

Em 1955, Garfield propôs um sistema bibliográfico que tinha por objetivo minimizar a citação de dados de má qualidade. O índice de citação reuniria todos os documentos que citaram ou criticaram determinado assunto da literatura científica (Garfield, 1955).

Atualmente, a preocupação com os atributos referentes à qualidade da informação tem ganhado mais um foco: a análise dos processos de produção, de disseminação e de recuperação dos dados, como iniciativa para garantir a usabilidade do conteúdo de pesquisas divulgadas (Sordi et al., 2008).

Por entender que existe uma série de fatores que orientam a escolha de um ou outro estudo, um número cada vez maior de pesquisadores começou a observar aspectos como autoridade do cientista, credibilidade dos dados, atualidade e aplicabilidade do método ao estabelecer as fontes de embasamento de seus estudos (Macedo; Pagano, 2011).

Nos estudos que necessitam realizar experiências ou replicar anteriores, a inquietação é ainda maior, já que qualquer ensaio inválido atrasa a solução dos experimentos. Como as pesquisas em Engenharia de Produção estão entre as que mais necessitam de experimentos válidos, é natural que haja receio quanto aos documentos divulgados, o que de maneira involuntária beneficia a reformulação de muitos dos processos biblioteconômicos (Leal, 2010).

Nesse sentido, a existência de pesquisas que mensurem e estabeleçam indicadores sobre os documentos mais citados contribui de forma concreta com as ações pela busca da qualidade informacional, por tratar-se de um campo de estudo em expansão e com necessidade de pesquisas atuais.

O estudo em questão apresenta os resultados de uma pesquisa descritiva, com abordagem quantitativa e estratégia de campo. Através da adaptação dos conceitos da análise de citação, observaram-se as referências listadas nos artigos das publicações regulares da revista "Gestão \& Produção", buscando a identificação, o mapeamento e a apuração quantitativa: a) dos itens listados em cada trabalho; b) da vida média da literatura; c) do idioma; d) da frequência de uso das referências e; e) da frente de pesquisa.

\section{Métodos}

Lançada em 1994, a revista "Gestão \& Produção" tem como missão "Ser um meio de divulgação de trabalhos originais ou que apresentem resultados de estudos e pesquisas na área de Engenharia de Produção" (Gestão \& Produção, 2011, p.1), fato que justifica sua escolha como objeto de uma análise bibliométrica. Até 2011, publicaram-se nove volumes regulares (1994-1996, 2000,2007-2011) e nove especiais (1997-1999 e 2001-2006), com a finalidade de atender a algumas subáreas da comunidade científica da Engenharia de Produção.

Por entender que as edições especiais são direcionadas a um tema específico e, por consequência, a autores específicos e/ou especializados, selecionaram-se para análise apenas as referências das edições regulares, no total de 10651 amostras.

Delineou-se o estudo ancorado na pesquisa descritiva com abordagem quantitativa (Rudio, 1978). Quanto aos procedimentos técnicos, adotou-se a estratégia documental na qual "[...] uma pesquisa se utiliza apenas de fontes documentais" (Appolinário, 2009, p.85) aplicadas a análise de citação (Gross; Gross, 1927; Garfield, 1955).

Para determinar o foco do trabalho, especificou-se: 1) como sujeitos da pesquisa os documentos referenciados nos artigos; 2) como variáveis os elementos determinísticos obtidos através da análise de referências; e 3) como tipo de recurso a pesquisa bibliográfica sobre o tema com vistas à construção do marco teórico e à compreensão da problemática abordada (Gil, 2002).

\section{Instrumentos}

Entendendo que uma base de dados sólida e confiável são alguns dos critérios para atestar a qualidade de uma pesquisa, adotou-se como fonte de informação a Scientific Eletronic Library Online (SciELO) Brasil, e, como aplicativos auxiliares, o Google Scholar e o software Publish orPerish.

Na fase da coleta, executou-se a criação de um banco de dados com as referências, categorizando-as de acordo com indicadores a serem analisados: 1) Indicador quantitativo de referências - número percentual de referências utilizadas por artigo; 2) Indicador quantitativo do 
idioma - estabelecimento do idioma que mais aparece nas referências; 3 ) Indicador quantitativo da vida média da literatura - identificação da cronologia das fontes de pesquisa; 4) Indicador quantitativo da frequência de uso das referências - estabelecimento da quantidade de vezes que os itens foram referenciados na publicação e; 5) Indicador quantitativo da frente de pesquisa - identificação dos documentos mais citados nos artigos publicados.

\section{Resultados e Discussão}

Quanto à apresentação e interpretação dos dados, adotou-se a representação conceitual, gráfica e numérica, de forma a permitir a visualização completa dos resultados observados, priorizando-se a análise dos principais resultados da pesquisa.

\section{Indicador quantitativo de referências utilizadas por artigo}

A Tabela 1 apresenta o número de artigos publicados nas edições regulares, o número e a média de referências por edição, considerando todos os seus tipos (Associação Brasileira de Normas Técnicas, 2002).

Com base nos dados, verifica-se que o número médio de referências listadas no periódico foi de 31,33. A maior média de trabalhos utilizados deu-se nas edições de 2007, correspondendo a 38,40; já a menor média foi de 13,25, nas edições lançadas em 1995.

Tabela 1. Número médio de referências por artigo.

\begin{tabular}{lccc}
\hline $\begin{array}{l}\text { Edições regulares } \\
\text { (ano) }\end{array}$ & $\begin{array}{c}\text { No de artigos } \\
\text { publicados }\end{array}$ & $\begin{array}{c}\text { No de } \\
\text { referências referências por artigo }\end{array}$ & $\begin{array}{c}\text { No médio de } \\
1994\end{array}$ \\
17 & 329 & 19,35 \\
1995 & 20 & 265 & 13,25 \\
1996 & 16 & 374 & 23,38 \\
2000 & 23 & 517 & 22,48 \\
2007 & 43 & 1651 & 38,40 \\
2008 & 46 & 1440 & 31,30 \\
2009 & 55 & 1760 & 32,00 \\
2010 & 60 & 2161 & 36,02 \\
2011 & 60 & 2154 & 35,90 \\
\hline Total & 340 & 10651 & 31,33 \\
\hline
\end{tabular}

Assim, se considerados Martins e Soma (2008), que apontam o uso médio de referências por artigo nas Engenharias em 26,45, os resultados demonstram que 31,33 está na média quantitativa do uso de documentos para embasar publicações dessa área.

O fato pode ser analisado sobre duas vertentes dicotômicas:

1) Partindo do conceito de que o uso de citações "[...] evidenciam elos entre indivíduos, instituições e áreas de pesquisa, visto que mostram o relacionamento de uma publicação com outra" (Foresti, 1989, p.3), há um baixo percentual de elo científico entre os autores e o estado da arte da área;

2) Os autores têm feito uso das referências listadas nos artigos apenas como um complemento para fornecer ao leitor mais informação acerca do assunto (Laloe; Mosseri, 2009), dando maior ênfase aos argumentos científicos esboçados e aos resultados obtidos. Ou, ainda, esses autores têm adotado menor número de referências, mas com alto fator de impacto e aceitação da comunidade científica (Volpato; Freitas, 2003).

\section{Indicador quantitativo do idioma}

Para o estabelecimento do indicador quantitativo do idioma, levaram-se em consideração a autoria, o idioma do título e os dados de publicação e verificou-se que 67,02\% das referências são estrangeiras: 65,29\% do Inglês, 0,80\% do Francês, 0,74\% do Espanhol e 0,19\% de outros idiomas. Conjectura-se que o uso de apenas 32,98 documentos em Português pode ser o reflexo da "crença" em uma maior qualidade das pesquisas estrangeiras em detrimento das nacionais (Volpato, 2005).

Ainda sobre a análise do idioma das referências, há de se levar em conta uma tendência de alguns estudiosos brasileiros em replicar apenas variações de experiências realizadas devido aos aspectos burocráticos do financiamento das pesquisas que dificultam a execução de trabalhos inovadores (Barata, 2010).

Indicador quantitativo de vida média da literatura

Para verificar a vida média da literatura (Burton; Kleber, 1960), estabeleceu-se a quantidade de referências 
Tabela 2. Distribuição da vida média das referências.

\begin{tabular}{lrr}
\hline \multirow{2}{*}{ Vida Média (em anos) } & \multicolumn{2}{c}{ Referências } \\
\cline { 2 - 3 } & $\mathrm{n}$ & \\
\hline Literatura $^{*}$ & 3262 & 30,67 \\
Atual $^{*}$ & 4970 & 46,73 \\
10 & 1719 & 16,16 \\
20 & 458 & 4,31 \\
30 & 138 & 1,30 \\
40 & 52 & 0,49 \\
50 & 19 & 0,18 \\
60 & 17 & 0,08 \\
mais de 60 & 10635 & 100,00 \\
\hline Total & &
\end{tabular}

*Década da edição e do documento iguais.

por década em cada edição e, na sequência, a diferença da relação temporal entre a data em que foi feita a referência (década de publicação do volume) e a data de publicação do documento referenciado (década da referência). Nesse processo, excluíram-se 16 amostras relativas a referências sem data.

Os resultados (Tabela 2) apontam o uso de 30,67\% de literatura atual, 46,73\% de literatura recente editada nos últimos 10 anos, 16,16\% editada nos últimos 20 anos e 22,52\% com mais de 20 anos de edição, partindo-se da data em que foi referenciado os artigos, confirma a tendência no uso de literatura recente e que, de certa forma, traduz os avanços científicos da área (Foresti, 1991).

Ainda sobre a literatura recente (46,73\%) aparecer como o maior índice de uso pelos pesquisadores, percebe-se que os dados vêm ao encontro de pesquisas que apontam que a área de Engenharia apresenta alta taxa (72,30\%) de trabalhos citados somente após quatro anos de sua publicação (Hamilton, 1991).

Além disso, identifica-se a frequência de uso de referências efêmeras (30,67\%) com média de vida curta por parte dos autores, o que, segundo Burton e Kleber (1960), é característico das áreas de Engenharia.

\section{Indicador quantitativo de frequência de uso das referências}

Através dos dados, identificam-se baixa influência e conexão entre os estudiosos (Chiari et al., 2007), já que 91,54\% dos documentos apareceram na lista de referências dos artigos apenas uma vez; 6,02\% apenas duas; 1,43\% somente três; e quatro ou mais referências surgem em menos de 1,00\% das listas.

Outro ponto relevante é que, por ser uma área muito abrangente e com possibilidade de enquadramento de suas publicações em diversos estratos (Lins; Pessoa, 2010), o pesquisador tem a seu favor a existência de uma variedade de fundamentações conceituais que embasam os trabalhos, o que também pode explicar a diversidade de documentos citados.

\section{Indicador quantitativo da frente de pesquisa}

A Tabela 3 possibilita diversas análises, entretanto optou-se pela verificação da frente de pesquisa referente aos estudos da área de Engenharia de Produção e de suas subáreas e seus aspectos qualitativos. Para tanto, apresentam-se: as obras mais citadas e a quantidade de citações no objeto de estudo, a análise sincrônica de citações em outras obras com as estimativas do Google Scholar e do Publish or Perish, a média geral de citação por ano e as variações na recuperação da obra.

Com vistas ao papel da referência dentro do trabalho científico (Noronha; Ferreira, 2000), a estimativa dos dados revela que as tendências epistemológicas dos artigos publicados baseiam-se: 1) principalmente nos conceitos abordados na obra Estudo de Caso: planejamento e métodos de R.K. Yin em suas diferentes edições e idiomas e 2) em teorias e métodos de estudos publicados em livros, já que 75\% dos itens que formaram a frente de pesquisa são compostos por esse tipo de documento.

Além disso, a análise sincrônica entre as estimativas do Google Scholar e do Publish or Perish confirmam as críticas ao uso dos índices de citações como um dos parâmetros para mensurar a produção científica dos autores (Adler et al., 2009), pois ambos os recursos analisam citações de documentos, e, o que é ainda mais grave, o Publish or Perish faz uso do Google Scholar para obter as citações primárias (Harzing, 2007), fato que deveria assegurar a igualdade dos dados. Entretanto, 65\% das referências analisadas apresentam quantidade do índice de citação em outras obras divergentes, e, dos 20 itens que compõem a frente de pesquisa, $80 \%$ apresentam variações no número de referências do mesmo material. 
Tabela 3. Frente de pesquisa dos autores mais influentes.

\begin{tabular}{|c|c|c|c|c|c|c|}
\hline \multirow{2}{*}{ Referências } & \multirow{2}{*}{$\begin{array}{l}\text { Refências no } \\
\text { periódico (n) }\end{array}$} & \multicolumn{2}{|c|}{ Referências no } & \multirow{2}{*}{$\begin{array}{c}\text { \# entre a } \\
\text { estimativa dos } \\
\text { softwares }\end{array}$} & \multirow{2}{*}{$\begin{array}{c}\text { Média de citações/ } \\
\text { ano (Publish or } \\
\text { Perish) }\end{array}$} & \multirow{2}{*}{$\begin{array}{c}\text { Variações de } \\
\text { referências do } \\
\text { mesmo materia }\end{array}$} \\
\hline & & Google Scholar & Publish or Perish & & & \\
\hline 1. Yin (2001) & 22 & 23 & 34 & 11 & 2,83 & 4 \\
\hline 2. Eisenhardt (1989) & 20 & 17002 & 17002 & 0 & 708,42 & 0 \\
\hline 3. Yin (2005) & 16 & 2 & 2 & 0 & 0,25 & 0 \\
\hline 4. Bryman (1989) & 13 & 1215 & 1217 & 2 & 0,13 & 3 \\
\hline 5. Rozenfeld et al. (2006) & 12 & 340 & 349 & 9 & 49,86 & 2 \\
\hline 6. Clark; Fujimoto (1991) & 11 & 3940 & 3933 & 7 & 178,77 & 8 \\
\hline 7. Prahalad; Hamel (1990) & 10 & 17028 & 84 & 16944 & 3,65 & 31 \\
\hline 8. Law; Kelton (1991) & 9 & 1275 & 22 & 1253 & 1,00 & 3 \\
\hline Malhotra (2001) & & 3261 & 32 & 3229 & 2,67 & 7 \\
\hline Yin (1994) & & 56787 & 12 & 56775 & 0,63 & 45 \\
\hline 9. Hax; Candea (1984) & 8 & 7 & 7 & 0 & 0,24 & 0 \\
\hline Kaplan; Norton (1997) & & 1664 & 1675 & 11 & 104,69 & 9 \\
\hline Slack (2002) & & 2313 & 26 & 2287 & 2,36 & 4 \\
\hline Voss et al. (2002) & & 916 & 916 & 0 & 83,27 & 19 \\
\hline 10. Coughlan; Coghlan (2002) & 7 & 427 & 427 & 0 & 38,32 & 16 \\
\hline Forza (2002) & & 310 & 310 & 0 & 28,18 & 16 \\
\hline Monden (1984) & & 139 & 139 & 0 & 4,79 & 0 \\
\hline Pires (2004) & & 200 & 202 & 2 & 22,44 & 4 \\
\hline Slack et al. (1997) & & 9 & 22 & 13 & 1,38 & 5 \\
\hline Wheelwright; Clark (1992) & & 2267 & 2494 & 227 & 118,76 & 9 \\
\hline
\end{tabular}

\section{Conclusão}

De forma geral, os resultados permitiram identificar os principais aspectos das fontes de pesquisas referentes às seguintes informações: vida média da literatura, idioma, frequência de uso das referências e frente de pesquisa. Tais informações fundamentam os trabalhos publicados na revista "Gestão \& Produção" e, por conseguinte, os trabalhos dos pesquisadores da comunidade científica da Engenharia de Produção e suas subáreas.

A primeira linha de resultados indica que, por um lado, os autores fazem uso de poucos trabalhos para referenciar suas publicações, o que pode ser percebido como uma preocupação em enfatizar muito mais o experimento e os resultados do que os conceitos existentes; e, por outro lado, há a falta de elo científico entre os pesquisadores da área.

A segunda linha demonstrou que há uma facilidade em acessar produções científicas internacionais, o que foi verificado pela predominância no uso de publicações de língua estrangeira, com grande parte concen- trando-se no idioma Inglês. O fato indica que os autores têm acompanhado os distintos aspectos dos experimentos científicos internacionais e/ou aqueles replicados em suas atividades científicas.

O terceiro resultado demonstra que os materiais atuais ou publicados nos últimos 10 anos compõem as fontes documentais que embasam os trabalhos, significando que os pesquisadores não detêm suas leituras apenas na literatura clássica, mas também nas definidas como efêmeras, o que, segundo estudos anteriores, caracteriza estudos na área de Engenharia.

Já o quarto apresenta uma variedade no uso das referências, representando uma diversidade na aplicação dos conceitos, métodos e experimentos nas pesquisas publicadas. $O$ último resultado identifica que grande parte dos autores que publicam no periódico baseiam-se nos trabalhos de R.K. Yin, além de embasar seus estudos, principalmente, em conceitos fornecidos no suporte livro.

Os resultados indicam a aplicação de outras análises bibliométricas ao objeto nos trabalhos posteriores, tal como a análise de conteúdos, além das sugestões 
finais apontadas nos artigos, ou seja, ampliar os debates sobre a validação dos indicadores de produção intelectual e padronização de critérios editoriais por área e realizar os estudos dos colégios invisíveis e a comparação do Core List da revista "Gestão \& Produção" com os que apresentam estratos elevado no Qualis.

\section{Referências}

ADLER, R.; EWING, J.; TAYLOR, P. Estatísticas de citações. Mediações, v.14, n.1, p.69-100, 2009.

APPOLINÁRIO, F. Dicionário de metodologia científica: um guia para a produção do conhecimento científico. São Paulo: Atlas, 2009

ASSOCIAÇÃO BRASILEIRA DE NORMAS TÉCNICAS. NBR 6023: informação e documentação: referências: elaboração. Rio de Janeiro: ABNT, 2002.

BARATA, G. A relação entre qualidade de artigos, ensino e carreira científica. Revista Eletrônica de Jornalismo Científico, 2010. Disponível em: <http://www.comciencia.br/ comciencia/?section $=3 \&$ noticia $=626>$. Acesso em: 16 maio 2012.

BRYMAN, A. Research methods and organization studies. London: Uniwin Hyman, 1989.

BURTON R.; KLEBER, R. The half-life of some scientific and technical literatures. American Documentation, v.11, n.1, p.18-22, 1960.

CHIARI, I.G. et al. As citações como base da rede social egocêntrica: o artigo citado e suas conexões. Marília: Unesp, 2007. Disponível em: <http://www.portalppgci.marilia.unesp.br>. Acesso em: 12 abr. 2012.

CLARK, K.B.; FUJIMOTO, T. Product development performance. Boston: Harvard Business School, 1991.

COUGHLAN, P.; COGHLAN, D. Action research for operations management. International Journal of Operations \& Production Management, v.22, n.2, p.220-240, 2002.

EISENHARDT, K. Building theories from case study research. Academy of Management Review, v.14, n.14, p.532-550, 1989.

FORESTI, N.A.B. Estudo da contribuição das revistas brasileiras de biblioteconomia e ciência da informação enquanto fonte de referência para a pesquisa. 1989. Dissertação (Mestrado) - Departamento de Biblioteconomia, Universidade de Brasília, Brasília, 1989.

FORESTI, N.A.B. Contribuição das revistas brasileiras de biblioteconomia e ciência da informação enquanto fonte de referência para a pesquisa. Ciência da Informação, v.19, n.1, p.53-71, 1991.

FORZA, C. Survey research in operations management: a process-based perspective. International Journal of Operations \& Production Management, v.22, n.2, p.152-194, 2002.

GARFIELD, E. Citation indexes for science: a new dimension in documentation through association of ideas. Science, v.122, n.3159, p.108-111, 1955. Available from: <http://www. garfield.library.upenn.edu/papers/science_v122v3159p 108y1955.html>. Cited: 21 Oct. 2011.
GESTÃO \& PRODUÇÃO. São Carlos: UFSCar, 1994-2011. Disponível em: <http://www.scielo.br>. Acesso em: 2 fev. 2012.

GIL, A.C. Como elaborar projetos de pesquisa. 4.ed. São Paulo: Atlas, 2002.

GROSS, P.L.K.; GROSS, E.M. College libraries and chemical education. Science, v.66, n.1723, p.385-389, 1927. Available from: <http://www.garfield.library.upenn.edu/papers/ grossandgross_science1927.pdf>. Cited: 18 Oct. 2011.

HAMILTON, D. Research papers: who's uncited now. Science, v.251, n.25, p.1410-1411, 1991.

HARZING, A.W. Publish or perish. 2007. Available from: <http:// www.harzing.com/pop.htm>. Cited: 5 Feb. 2012.

HAX, A.C.; CANDEA, D. Production and inventory management. Englewood Cliffs: Prentice-Hall, 1984.

KAPLAN, R.S.; NORTON, D.P. Estratégia em ação. Rio de Janeiro: Campus, 1997.

LALOE, F.; MOSSERI, R. Avaliação bibliométrica de pesquisadores: não é correta nem mesmo errada. Boletim da Sociedade Brasileira de Física, n.40, p.1-6, 2009.

LAW, A.M.; KELTON, W.D. Simulation modeling and analysis. $2^{\text {nd }}$. ed. New York: McGraw-Hill, 1991

$L E A L$, J. Reengenharia em bibliotecas. Revista Digital de Biblioteconomiae Ciência da Informação, v.8, n.1, p.12-20, 2010. Disponível em: <http://www.sbu.unicamp.br/seer/ojs/ index.php/sbu_rci/article/view/463>. Acesso em: 28 abr. 2012.

LINS, M.P.E.; PESSOA, L.A.M. Desafios da avaliação de publicações em periódicos: discutindo o novo Qualis da área engenharias III. RBPG, v.7, n.12, p.14-33, 2010.

MACEDO, T.S.; PAGANO, A.S. Análise de citações em textos acadêmicos escritos. DELTA, v.27, n.2, p.257-288, 2011. Disponível em: <http://www.scielo.br>. Acesso em: 7 mar. 2012.

MALHOTRA, N.K. Pesquisa de marketing. 3.ed. Porto Alegre: Bookman, 2001.

MARTINS, C.B.B.; SOMA, N.Y. O comportamento das áreas do conhecimento em termos de citações de artigos. In: ENCONTRO DE INICIAÇÃO CIENTÍFICA E PÓS-GRADUAÇÃO DO ITA, 14., 2008, São José dos Campos. Anais eletrônicos... São José dos Campos: ITA, 2008. Disponível em: <www.bibl.ita. br/xivencita/com09.pdf>.

MONDEN, Y. Sistema Toyota de produção. São Paulo: IMAM, 1984.

NORONHA, D.P.; FERREIRA, S.M.S.P. Índices de citação. In: CAMPELLO, B.S.; CENDÓN, B.V.; KREMER, J.M. (Org.). Fontes de 
informação para pesquisadores e profissionais. Belo Horizonte: UFMG, 2000. p.249-262.

PIRES, S.R.I. Gestão da cadeia de suprimentos. São Paulo: Atlas, 2004.

PRAHALAD, C.K.; HAMEL, G. The core competence of the corporation. Harvard Business Review, v.68, n.3, p.79-91, 1990.

ROZENFELD, H. et al. Gestão de desenvolvimento de produto. São Paulo: Saraiva, 2006.

RUDIO, F.V. Introdução ao projeto de pesquisa científica. Petrópolis: Vozes, 1978.

SLACK, N. et al. Administração da produção. São Paulo: Atlas, 1997.

SLACK, N. et al. Administração da produção. 2.ed. São Paulo: Atlas, 2002.

SORDI, J.O.; MEIRELES, M.; GRIJO, R.N. Gestão da qualidade da informação no contexto das organizações: percepções a partir do experimento de análise de confiabilidade dos jornais eletrônicos. Perspectivas em Ciência da Informação, v.13, n.2, p.168-195, 2008. Disponível em: <http://www.scielo.br>. Acesso em: 18 out. 2011.
VOLPATO, G.L. Publicação científica: desmistificando o drama. In: ENCONTRO SOBRE METODOLOGIA CIENTÍFICA, 2., 2005, Piracicaba. Anais... Piracicaba: ESALQ, 2005. Disponível em: <http://www.gilsonvolpato.com.br/pdf/2005\%20Volpato \%20-\%20Esalq\%20-\%20Publ\%20Cient\%20desmistificando\% 200\%20drama.pdf>. Acesso em: 18 out. 2011.

VOLPATO, G.L.; FREITAS, E.G. Desafios na publicação científica. Pesquisa Odontológica Brasileira, v.17, p.49-56, 2003.

VOSS, C.; TSIKRIKTSIS, N.; FROHLICH, M. Case research in operations management. International Journal of Operations \& Production Management, v.22, n.2, p.195-219, 2002.

WHEELWRIGHT, S.C.; CLARK, K.B. Revolutionizing product development: quantum leaps in speed, efficiency, and quality. New York: The Free, 1992.

YIN, R.K. Case study research: design and methods. $2^{\text {nd }}$.ed. London: Sage, 1994.

YIN, R.K. Estudo de caso: planejamento e métodos. 2.ed. Porto Alegre: Bookman, 2001.

YIN, R.K. Estudo de caso: planejamento e métodos. 3.ed. Porto Alegre: Bookman, 2005. 
